\title{
Single-layer economic model predictive control for periodic operation
}

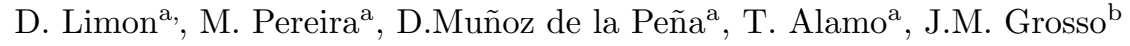 \\ ${ }^{a}$ Departamento de Ingeniería de Sistemas y Automática, Escuela Superior de Ingenieros, \\ Universidad de Sevilla. Sevilla, Spain. (e-mail: \{dlm,mpereiram,dmunoz,talamo\}@us.es) \\ ${ }^{b}$ Advanced Control Systems Research Group (SAC), at the Institut de Robotica $i$ \\ Informatica Industrial (CSIC-UPC). Barcelona (e-mail: \{jgrosso\}@iri.upc.es)
}

\begin{abstract}
In this paper we consider periodic optimal operation of constrained periodic linear systems. We propose an economic model predictive controller based on a single layer that unites dynamic real time optimization and control. The proposed controller guarantees closed-loop convergence to the optimal periodic trajectory that minimizes the average operation cost for a given economic criterion. A-priori calculation of the optimal trajectory is not required and if the economic cost function is changed, recursive feasibility and convergence to the new periodic optimal trajectory is guaranteed. The results are demonstrated with two simulation examples, a four tank system, and a simplified model of a section of Barcelona's water distribution network.
\end{abstract}

Keywords: Model predictive control; Economic MPC; Dynamic real time optimization; Periodic operation; Stability; Changing criteria.

\section{Introduction}

The main objective of a process control system is to guarantee closed-loop stability satisfying the process constraints while minimizing the operation cost. Model predictive control (MPC) has demonstrated to be a suitable control tech-

5 nique for optimal process operation thanks to its ability to yield high performance control systems capable of operating multivariable constrained systems without expert intervention for long periods of time [5, 24].

Most industrial processes are typically operated at the equilibrium point that minimizes an economic cost function. Standard MPC approaches are based on

10 a hierarchical structure in which the operation point is calculated by a real-time optimizer (RTO), and then a model predictive controller is designed to drive the system to this operation point [6, 7]. In order to enhance the performance during the transient of the system, economic MPC has been proposed [3, 22. The main characteristic of this predictive controller is that the MPC cost function uses the 15 economic cost function as stage cost function. This allows the controller to take into account the performance during the transient but brings stability issues.

Preprint submitted to Journal of $E^{A} T_{E} X$ Templates 
In 10, 11, 12, Lyapunov based model predictive control designs, which are capable of optimizing closed-loop performance with respect to general economic considerations for a broad class of nonlinear process systems, including systems 20 subject to asynchronous and delayed measurements and uncertain variables; have been developed. The proposed techniques are based on two different modes of operation that guarantee that the closed-loop system is ultimately bounded in a small region containing the origin.

In [23, recent results on the stabilizing design of economic MPC are sum25 marized. In [9] an stabilizing economic MPC without terminal constraint is presented. In 28] a single layer economic MPC has been proposed by integrating the RTO into the MPC as terminal cost function and the benefits of this controller has been practically validated.

It has been proved that steady state operation results to be optimal when 30 the system is strictly dissipative with respect to the economic cost function [2]. However in certain cases, the optimal operation of the plant from an economic point of view is not to remain at a given steady state but to follow a nonsteady trajectory, often periodical [17, 14]. This is the case for instance when the system is subject to periodic disturbances (such as an exogenous periodic

35 demand of water distribution networks or supply chains), fluctuating prices of the economic cost function (such as the electricity unitary cost), or time varying dynamics (such as batch nonlinear-process operation). In [13] it is reported that processes such as simulated moving bed (SMB) and pressure swing adsorption (PSA) present a better economic performance if they are operated following a 40 periodic trajectory.

To deal with non steady periodic operation of a plant, the predictive control structure must be modified. One solution proposed in the literature is to follow a two layer approach in which the optimal periodic trajectory is calculated by a dynamic real time optimizer (DRTO), which takes into account a dynamic model 45 of the plant; and based on this optimal periodic trajectory, a model predictive controller for tracking the optimal trajectory is applied, see for example [27. If the model predictive controller is designed appropriately [24, asymptotic convergence of the closed-loop system to the optimal trajectory can be proved. In order to improve the economic performance during the transient, several ${ }_{50}$ authors propose to use economic MPC to track the optimal trajectory. In 2 an economic MPC that guarantees that the asymptotic average economic cost of the controlled system is no worse that the average economic cost of the optimal trajectory has been presented. In [14] an economic MPC for cyclic processes is presented. Lyapunov stability of the controlled plant is derived if the initial state 55 is in a neighborhood of the optimal trajectory. In [13] stability and robustness of infinite horizon economic MPC is analyzed.

In general, all the above mentioned control strategies require the calculation of the optimal periodic trajectory by the real time optimization layer for the given economic cost function. The economic cost function typically depends on 60 exogenous parameters, such as unitary prices or expected demands, that may be changed throughout the operation of the plant. When these parameters are changed, then the optimal trajectory must be recalculated and the predictive 
controllers should be re-designed to this new scenario by adapting the constraints and/or the cost function appropriately. The subsequent variation of the constraints of the optimization problem could lead to feasibility loss 20,8 .

Motivated by these issues, in this paper we consider periodic optimal operation of constrained periodic linear systems. We propose an economic model predictive controller based on a single layer that unites dynamic real time optimization and control following the idea of [28]. The proposed controller guaran-

70 tees closed-loop convergence to the optimal periodic trajectory that minimizes average operation cost for a given economic criterion. A-priori calculation of this optimal trajectory is not required. In addition, if the economic cost function is changed, recursive feasibility and convergence to the new periodic optimal trajectory is guaranteed. The results are demonstrated with two simulation ex75 amples, a four tank system, and a simplified model of a section of Barcelona's water distribution network.

\section{Notation}

Bold letters are used to denote the sequence of $T$ values of the signal, i.e. $\mathbf{z}_{T}=\{z(0), \cdots, z(T-1)\} . \mathbf{z}_{T}(k)$ denotes the sequence $\mathbf{z}(k)=\{z(k), \cdots, z(k+$

so $T-1)\}$; if the sequence depends on a parameter $\theta$, then this is denoted as $\mathbf{z}(k ; \theta)=\{z(k ; \theta), \cdots, z(k+T-1 ; \theta)\} . \mathbb{I}_{[a, b]}$ denotes the set of integer numbers contained in the interval $[a, b]$, that is $\mathbb{I}_{[a, b]}=\{a, a+1, \cdots, b\}$. For a certain parametric optimization problem $\min _{z \in Z} F(z ; \theta)$, the optimal solution is denoted as $z^{o}(\theta)$.

\section{Problem formulation}

In this work we focus on the following class of time-varying linear systems

$$
x(k+1)=A(k) x(k)+B(k) u(k)+w(k)
$$

where $x(k) \in \mathbb{R}^{n}, u(k) \in \mathbb{R}^{m}$ and $w(k) \in \mathbb{R}^{n}$ are the state, input and disturbance vectors of the system at time step $k$ respectively. The evolution of the matrices $A(k)$ and $B(k)$ as well as the disturbance signal $w(k)$ are known.

The dynamic model can be considered as a time varying affine system denoted as

$$
x(k+1)=f(k, x(k), u(k))
$$

with $f(k, x, u)=A(k) x+B(k) u+w(k)$.

The state and input must satisfy the following constraints

$$
(x(k), u(k)) \in \mathcal{Z}(k) \subseteq \mathbb{R}^{n+m}
$$

where $\mathcal{Z}(k)$ is a closed convex polyhedron that may vary in time. It is assumed that $\mathcal{Z}(k)$ is known and contains the origin in its interior.

The performance of the evolution of the plant is measured by an economic stage cost function $\ell(k, x, u, p)$ that depends on the current state and input of the plant, on the time and on a exogenous parameter $p$. The value of the parameter 
$p$ may be changed during the operation of the plant and this variation is not known a priori. This function is assumed to be positive $\ell(k, x, u, p) \geq 0$ for all $(k, x, u, p)$ and convex in $(x, u)$ for all $k$ and $p$.

100 Remark 1. No assumption is made on how the parameter $p$ affects the cost function. It can be a numerical parameter that can be changed (as for instance unitary prices) or a switch between completely different and unrelated economic cost functions. The policy of these changes is not known and then the future evolution of $\mathrm{p}$ is uncertain. The variations of $p$ may induce a dramatic variation on 105 the optimal operation of the system. This is shown in the illustrative examples. A sudden change in $p$ is equivalent to a set-point change for a standard MPC for regulation.

In this paper we focus on periodic operation of a closed-loop system with a fixed period $T$. The periodic behavior may be a consequence of the time-varying system dynamics, the exogenous disturbances, the constraints and/or the time varying economic stage cost function. Thus, these functions are considered to be periodic, as it is stated in the following assumption.

Assumption 1. The system is periodic and its period is $T$. That is, for all $k$, the following equations hold

$$
\begin{aligned}
A(k) & =A(k+T) \\
B(k) & =B(k+T) \\
w(k) & =w(k+T) \\
\mathcal{Z}(k) & =\mathcal{Z}(k+T) \\
\ell(k, x, u, p) & =\ell(k+T, x, u, p), \quad \forall(x, u) \in \mathcal{Z}(k), \forall p
\end{aligned}
$$

\subsection{Economically optimal periodic operation}

The main objective of the proposed control system is to operate the plant to achieve an optimal economic performance. The economic performance is measured with the average of the economic cost function of the closed-loop system trajectories, that is

$$
L_{\infty}\left(0, x(0), \mathbf{u}_{\infty}(0), p\right)=\lim _{M \rightarrow \infty} \frac{1}{M} \sum_{j=0}^{M-1} \ell(j, x(j), u(j), p)
$$

where $x(0)$ is the initial state and $\mathbf{u}_{\infty}(0)$ its corresponding closed-loop input trajectories.

The optimal trajectory in which the system can be operated $\left(\mathbf{x}_{\infty}^{\star}, \mathbf{u}_{\infty}^{\star}\right)$ is derived from the solution of the following optimization problem in which the initial state is a free variable

$$
\begin{aligned}
\min _{x(0), \mathbf{u}_{\infty}} & L_{\infty}\left(0, x(0), \mathbf{u}_{\infty}, p\right) \\
\text { s.t. } & x(j+1)=f(j, x(j), u(j)) \\
& (x(j), u(j)) \in \mathcal{Z}_{r}(j), \quad \forall j \geq 0
\end{aligned}
$$


where the set $\mathcal{Z}_{r}(j)$ is a closed polyhedron contained into the relative interior of $\mathcal{Z}(j)$ in order to ensure that the constraint $\mathcal{Z}(j)$ is not active in the optimal trajectory

The optimal state and input trajectories are $\mathbf{x}_{\infty}^{\star}$ and $\mathbf{u}_{\infty}^{\star}$ respectively. It is assumed that the solution of the optimization problem (4), an therefore the optimal trajectory, is unique for a given $p$. The optimal economic cost function is denoted as $L_{\infty}^{\star}(p)$. Notice that these optimal trajectories depend on the value of the exogenous parameter $p$. This dependence will be emphasized by denoting the optimal trajectories as $\mathbf{x}_{\infty}^{\star}(p)$ and $\mathbf{u}_{\infty}^{\star}(p)$.

In general, problem (4) has an infinite number of decision variables, however given the periodic nature of the dynamics, the constraints and the cost function, under certain assumptions of uniqueness of the solution, the optimal trajectories can be obtained solving a finite horizon open-loop problem that optimizes the average cost of a period [2]. In particular, the optimal solution can be obtained from the solution of the following optimization problem at any given time instant $k$, which we denote as $P_{D R T O}(k, p)$ :

$$
\begin{aligned}
\mathcal{L}_{T}^{o}(k, p)=\min _{x(0), \mathbf{u}_{T}} & \sum_{j=0}^{T-1} \ell(k+j, x(j), u(j), p) \\
\text { s.t. } & x(j+1)=f(k+j, x(j), u(j)) \\
& (x(j), u(j)) \in \mathcal{Z}(k+j), j \in \mathbb{I}_{[0, T-1]} \\
& x(0)=x(T)
\end{aligned}
$$

This is the so-called dynamic real time optimization, used in practice to adapt the optimal trajectories to possible variations on exogenous signals such as $p$.

Theorem 1. The optimal solution $P_{D R T O}(k, p)$ satisfies the following equations

$$
\begin{aligned}
x^{o}(j ; k) & =x^{\star}(k+j) \\
u^{o}(j ; k) & =u^{\star}(k+j) \\
\mathcal{L}_{T}^{o}(k, p) & =\mathcal{L}_{T}^{o}(0, p)=T L_{\infty}^{\star}(p)
\end{aligned}
$$

Proof. Assuming that $k \in \mathbb{I}_{[0, T-1]}$, then the solution $x(0 ; k)=x^{o}(1 ; k-1)$ and $u(j ; k)=u^{o}(j+1 ; k-1)$ for $j \in \mathbb{I}_{[0, T-2]}$ and $u(T-1 ; k)=u^{o}(0 ; k-1)$ is a feasible solution of $P_{D R T O}(k, p)$. The cost function of this feasible solution is equal to the optimal cost function of $P_{D R T O}(k-1, p), \mathcal{L}_{T}^{o}(k-1, p)$, and then, from optimality we have that $\mathcal{L}_{T}^{o}(k, p) \leq \mathcal{L}_{T}^{o}(k-1, p)$. Then it holds that

$$
\mathcal{L}_{T}^{o}(T, p) \leq \mathcal{L}_{T}^{o}(k, p) \leq \mathcal{L}_{T}^{o}(k-1, p) \leq \mathcal{L}_{T}^{o}(0, p)
$$

Since the system is periodic, $\mathcal{L}_{T}^{o}(0, p)=\mathcal{L}_{T}^{o}(T, p)$ and $\mathcal{L}_{T}^{o}(k, p)=\mathcal{L}_{T}^{o}(0, p)$ for all $k$.

\footnotetext{
${ }^{1}$ This tighter set of constraints is added to avoid the possible loss of controllability due the existence of active constraints. This is not a limitation from a practical point of view since this set can be chosen arbitrarily close to the real constraint set.
} 
The infinite horizon optimal trajectory $\left(\mathbf{x}_{\infty}^{\star}, \mathbf{u}_{\infty}^{\star}\right)$ is obtained extending the solution obtained periodically.

In general, the initial state of the system will be different to the corresponding state of the optimal trajectory. The control objective is to derive a control law $u(k)=\kappa(k, x(k), p)$ such that the evolution of the closed-loop system

$$
x(k+1)=f(k, x(k), \kappa(k, x(k), p))
$$

fulfils the constraints (3) and the average economic performance is asymptotically minimized, that is, $(x(k), u(k))$ converges to $\left(x^{\star}(k ; p), u^{\star}(k ; p)\right)$.

\section{Economic MPC for changing periodic operation}

The proposed predictive controller combines the dynamic real time optimization and the control decision in a single optimization problem. To this aim, an artificial periodic trajectory is added as a new decision variable. The cost function is defined as the sum of two terms: (i) a tracking term that penalizes the deviation between the predicted trajectory and the artificial periodic trajectory and (ii) an economic term that measures the economic cost function of the artificial periodic trajectory. The cost function of the controller optimization problem is defined as follows:

$$
\begin{aligned}
V_{N}\left(k, x, p, \overline{\mathbf{u}}_{N}, x^{a}(0), \mathbf{u}_{T}^{a}\right)= & \sum_{i=0}^{N-1}\left\|\bar{x}(i)-x^{a}(i)\right\|_{Q}^{2}+\left\|\bar{u}(i)-u^{a}(i)\right\|_{R}^{2} \\
& +\sum_{j=0}^{T-1} \ell\left(k+j, x^{a}(j), u^{a}(j), p\right)
\end{aligned}
$$

where $N$ is the prediction horizon and $T$ is the period of the system. It is assumed that $N \leq T$.

The control law is derived from the solution of following optimization problem $P_{N}(k, x, p)$ :

$$
\begin{aligned}
\min _{\overline{\mathbf{u}}_{N}, x^{a}(0), \mathbf{u}_{T}^{a}} & V_{N}\left(k, x, p, \overline{\mathbf{u}}_{N}, x^{a}(0), \mathbf{u}_{T}^{a}\right) \\
\text { s.t. } & \bar{x}(0)=x \\
& \bar{x}(i+1)=f(k+i, \bar{x}(i), \bar{u}(i)) \\
& (\bar{x}(i), \bar{u}(i)) \in \mathcal{Z}(k+i), \quad i \in \mathbb{I}_{[0, N-1]} \\
& x^{a}(j+1)=f\left(k+j, x^{a}(j), u^{a}(j)\right) \\
& \left(x^{a}(j), u^{a}(j)\right) \in \mathcal{Z}^{r}(k+j), j \in \mathbb{I}_{[0, T-1]} \\
& x^{a}(0)=x^{a}(T) \\
& \bar{x}(N)=x^{a}(N)
\end{aligned}
$$

where $\overline{\mathbf{u}}_{N}$ is the predicted input trajectory and $x_{0}^{a}, \mathbf{u}_{T}^{a}$ are the artificial initial state and input trajectories respectively. 
Constraints (6b)-(6d) guarantee that the predicted trajectory of the system is admissible. The set of constraints on the artificial periodic trajectory (6e)(6g) are identical to the constraints of the DRTO optimization problem (5), and guarantee that this trajectory is admissible and periodic. The terminal constraint $6 \mathrm{~h}$ ) forces the predicted trajectory to reach the artificial trajectory in $N$ steps.

$P_{N}(k, x, p)$ is a convex optimization problem in which the cost function is convex and the constraints are linear. This problem can be efficiently solved using specialized algorithms such as interior point methods 44. The optimal solution is denoted $\left(\overline{\mathbf{u}}_{N}^{o}, x^{a o}(0), \mathbf{u}_{T}^{a o}\right)$ and it is assumed to be unique for a given 175 . The proposed control law is derived from the receding horizon policy

$$
u(k)=\bar{u}^{o}(0)=\kappa(k, x, p)
$$

The set of constraints (6b)- $6 \mathrm{~h}$ ) does not depend on the exogenous parameter $p$. This implies that a change of the value of $p$ cannot cause a loss of feasibility of the optimization problem. Indeed, a change on the economic stage cost function affects only the cost function of the optimization problem. The set of states where the optimization problem $P_{N}(k, x, p)$ is feasible is denoted as $X_{N}(k)$. The set of feasible initial states $X_{N}(0)$ will be denoted as $X_{N}$.

We will prove next the following properties of the proposed controller: (i) if the initial state is such that $P_{N}(0, x(0), p(0))$ is feasible, then all the subsequent optimization problems $P_{N}(k, x(k), p(k))$ will be feasible even in the case that 185 the parameter $p$ is changed and (ii) the optimal trajectory is an asymptotically stable trajectory of the closed-loop system in the Lyapunov sense.

Before stating the main theorem of the paper the notion of stability is introduced and a Lypaunov sufficient condition is shown.

\subsection{Lyapunov asymptotic stability of a periodic trajectory}

Consider a closed-loop system given by

$$
x(k+1)=f(k, x(k), \kappa(k, x(k)))=f_{\kappa}(k, x(k))
$$

subject to the constraints $x(k) \in \mathcal{X}_{\kappa}(k)=\{x:(x, \kappa(x)) \in \mathcal{Z}\}$. Assume that the trajectory $x_{t}(k)$ is a trajectory of system (8) such that $x_{t}(k) \in X_{\kappa r}(k)$, where $X_{\kappa r}(k)$ is the relative interior of $X_{\kappa}(k)$.

In this paper a Lyapunov stability notion is adopted: the state $x(k)$ converges to the optimal trajectory $x_{t}(k)$ and, near the optimal trajectory, small changes in the initial state $x(0)$ cause small changes in the resulting trajectory. Denoting the tracking error as follows

$$
e(k)=x(k)-x_{t}(k),
$$

the trajectory $x_{t}(k)$ is an asymptotic stable trajectory of the system if there exists a set of initial states $\Gamma$ and a $\mathcal{K} \mathcal{L}$ function $2, \beta(\cdot, \cdot)$, such that for all

\footnotetext{
${ }^{2} \mathrm{~A}$ function $\beta(s, t)$ is a $\mathcal{K} \mathcal{L}$ function if $\beta(\cdot, t)$ is a $\mathcal{K}_{\infty}$ function for all $t \geq 0$ and if $\beta(s, \cdot)$ is strictly decreasing converging to zero for all $s>0$.
} 
$x(0) \in \Gamma$, then

$$
\|e(k)\| \leq \beta(\|e(0)\|, k)
$$

and $x(k) \in X_{\kappa}(k)$ for all $k$.

Asymptotic stability can be proved by the conditions of the following theorem.

Theorem 2. Let $\{\Omega(\cdot)\}$ be a sequence of closed sets that may be time varying, such that for all $k \geq 0$, (i) $x_{t}(k)$ is in the interior of $\Omega(k)$, (ii) $\Omega(k) \subseteq X_{\kappa}(k)$ and (iii) $f_{\kappa}(k, x(k)) \in \Omega(k+1)$ for all $x(k) \in \Omega(k)$. Assume that there exists a continuous function $W(k, x): \mathbb{R} \times \mathbb{R}^{n} \rightarrow \mathbb{R}$ such that

$$
\begin{aligned}
W(k, x(k)) & \geq \alpha_{1}(\|e(k)\|), \quad \forall x(k) \in \Omega(k) \\
W(k, x(k)) & \leq \alpha_{2}(\|e(k)\|), \quad \forall e(k) \in B_{r} \\
W(k+1, x(k+1))-W(k, x(k)) & \leq-\alpha_{3}(\|e(k)\|) \quad \forall x(k) \in \Omega(k)
\end{aligned}
$$

200 $\|z\| \leq r\}$. Then $x_{t}(k)$ is an asymptotic stable trajectory of the system (8) for all $x(0) \in \Omega(0)$.

Proof. Since $W(k, x)$ is continuous and $x_{t}(k)$ is contained in the interior of $\Omega(k)$, by means of Proposition 2 of the postface to the book [24], there exists a $\mathcal{K}_{\infty}$ function $\tilde{\alpha}_{2}(\cdot)$ such that

$$
W(k, x(k)) \leq \tilde{\alpha}_{2}(\|e(k)\|), \quad \forall x(k) \in \Omega(k)
$$

Notice that this implies that $\|e(k)\| \geq \tilde{\alpha}_{2}^{-1}(W(k, x(k)))$ and then

$$
\begin{aligned}
W(k+1, x(k+1)) & \leq W(k, x(k))-\alpha_{3}\left(\tilde{\alpha}_{2}^{-1}(W(k, x(k)))\right) \\
& \leq \phi(W(k, x(k))
\end{aligned}
$$

where $\phi(\cdot)$ is a certain $\mathcal{K}_{\infty}$ function such that $\phi(s)<s$ for all $s>0$ [15]. From this inequality we have that

$$
\alpha_{1}(\|e(k)\|) \leq W(k, x(k)) \leq \phi^{k}(W(0, x(0))) \leq \phi^{k}\left(\tilde{\alpha}_{2}(\|e(0)\|)\right.
$$

where $\phi^{k}(s)$ is the $k$-th composition of function $\phi(\cdot)$, that is, $\phi^{k}(s)=\phi^{k-1}(\phi(s))$ with $\phi^{0}(s)=s$.

Taking into account that $\beta(s, t)=\alpha_{1}^{-1}\left(\phi^{t}\left(\tilde{\alpha}_{2}(s)\right)\right)$ is a $\mathcal{K} \mathcal{L}$ function [19], then

$$
\|e(k)\| \leq \beta(\|e(0)\|, k)
$$

for all $x(0) \in \Omega(0)$. On the other hand, by definition $x(k) \in \Omega(k) \subseteq X_{\kappa}(k)$.

Once the stability framework is defined, then the stability properties of the proposed controller are stated in the following section. 


\subsection{Stabilizing design of the proposed controller}

210 will be proved defining a function based on the optimal cost function $V_{N}^{o}(k, x, p)$ of problem $P_{N}(k, x, p)$ that serves as Lyapunov function. To this aim, the following controllability condition on the system is assumed.

Assumption 2. For integers $0 \leq i \leq j \leq T$, we define the following function

$$
\Psi(j, i)=A(j) \cdot A(j-1) \cdots A(i+1) B(i) .
$$

Then, it is assumed that there exists an integer $n_{c}$ such that the matrix

$$
\left[\Psi\left(n_{c}-1,0\right), \cdots, \Psi\left(n_{c}-1, n_{c}-2\right), B\left(n_{c}-1\right)\right]
$$

is full row rank.

Furthermore, it is necessary to remark that in virtue of theorem C.34 in 24 the optimal cost function $V_{N}^{o}(k, x, p)$ is a continuous function in $x$.

We present next the main theorem of the paper:

Theorem 3. Let $\mathbf{x}_{\infty}^{\star}(p)$ and $\mathbf{u}_{\infty}^{\star}(p)$ be the economically optimal trajectory of the system derived from (4), let the prediction horizon $N$ be equal to or larger than the integer $n_{c}$ defined in assumption 2 and let $Q$ be a positive definite function, then the system controlled by the proposed control law is recursively feasible and the optimal trajectory is asymptotic stable for all feasible initial state, i.e. $x(0) \in X_{N}$.

ProOF. First it will be proved that the optimization problem is recursively feasible and then the convergence of the closed-loop system will be shown.

Feasibility will be proved by recursion. Consider that the optimization problem is feasible at time instant $k$, i.e. $x(k) \in X_{N}(k)$. Let $\overline{\mathbf{u}}_{N}^{o}(k), x_{0}^{a o}(k)$ and $\mathbf{u}_{T}^{a o}(k)$ be the optimal solution at sample $k$ and let define the following solutions for the next sampling time $k+1$,

$$
\begin{aligned}
\overline{\mathbf{u}}_{N}(k+1) & =\left(u^{o}(1 ; k), \cdots, u^{o}(N-1 ; k), u^{a o}(N ; k)\right) \\
x^{a}(0 ; k+1) & =x^{a o}(1 ; k) \\
\mathbf{u}_{T}^{a}(k+1) & =\left(u^{a o}(1 ; k), \cdots, u^{a o}(T-1 ; k), u^{a o}(0 ; k)\right) .
\end{aligned}
$$

${ }_{230}$ Taking into account that $\bar{x}^{o}(N ; k)=x^{a o}(N ; k)$, that the artificial trajectory is periodic, i.e. $x^{a o}(0 ; k)=x^{a o}(T ; k)$ and considering that $x(k+1)=\bar{x}^{o}(1 ; k)$, then the predicted trajectories are

$$
\begin{aligned}
\overline{\mathbf{x}}_{N+1}(k+1) & =\left(\bar{x}^{o}(1 ; k), \cdots, \bar{x}^{o}(N-1 ; k), x^{a o}(N ; k), x^{a o}(N+1 ; k)\right) \\
\mathbf{x}_{T+1}^{a}(k+1) & =\left(x^{a o}(1 ; k), \cdots, x^{a o}(T-1 ; k), x^{a o}(0 ; k), x^{a o}(1 ; k)\right)
\end{aligned}
$$

From the definition of the optimization problem constraints and taking into account that the optimal solution at time $k$ is feasible, it can be proved that 
the aforementioned defined trajectory is feasible for time step $k+1$, and hence that $x(k+1) \in X_{N}(k+1)$.

Taking into account that $x(0) \in X_{N}$, this implies that $x(k) \in X_{N}(k)$ for all $k$.

Asymptotic stability will be proved demonstrating that the function

$$
W(k, x)=V_{N}^{o}(k, x, p)-T L_{\infty}^{\star}(p)
$$

is a Lyapunov function for the optimal trajectory $\mathbf{x}_{\infty}^{\star}(p)$ and satisfies the as240 sumptions of Theorem 2 .

Defining the error signal as

$$
e(k)=x(k)-x^{\star}(k ; p)
$$

the following statements are proved: (1) $W(k, x(k)) \geq \alpha_{1}(\|e(k)\|)$ for all $x(k) \in$ $X_{N}(k)$ :

From the definition of the Lyapunov function we have that

$$
\begin{aligned}
W(k, x(k)) \geq \quad & \left\|x(k)-x^{a o}(0 ; k)\right\|_{Q}^{2} \\
& +T L_{T}\left(k, x^{a o}(0 ; k), \mathbf{u}_{T}^{a o}(k), p\right)-T L_{\infty}(p)
\end{aligned}
$$

245 From the convexity and uniqueness of $P_{D R T O}(k, p)$ we have have that there exists a $\mathcal{K}_{\infty}$ function $\pi_{1}(\cdot)$ such that

$$
\begin{aligned}
L_{T}\left(k, x^{a o}(0 ; k), \mathbf{u}_{T}^{a o}(k), p\right) \geq & L_{\infty}(p) \\
& +\pi_{1}\left(\left\|x^{a o}(0 ; k)-x^{\star}(k ; p)\right\|\right)
\end{aligned}
$$

and then in virtue of the properties of the $\mathcal{K}$ functions we have that

$$
\begin{aligned}
W(k, x(k)) \geq & \left\|x(k)-x^{a o}(0 ; k)\right\|_{Q}^{2} \\
& +T \pi_{1}\left(\left\|x^{a o}(0 ; k)-x^{\star}(k ; p)\right\|\right) \\
\geq & \alpha_{1}\left(\left\|x(k)-x^{a o}(0 ; k)\right\|+\left\|x^{a o}(0 ; k)-x^{\star}(k ; p)\right\|\right) \\
\geq & \alpha_{1}\left(\left\|x(k)-x^{\star}(k ; p)\right\|\right)
\end{aligned}
$$

for a some $\mathcal{K}_{\infty}$ function $\alpha_{1}(\cdot)$.

250

(2) $W(k, x(k)) \leq \alpha_{2}(\|e(k)\|)$ for all $e(k) \in B_{\epsilon}$ :

From the lemma 3 we have that $W(k, x(k)) \leq \alpha_{2}\left(\left\|x(k)-x^{\star}(k ; p)\right\|\right)$ for all $\left\|x(k)-x^{\star}(k ; p)\right\| \leq \varepsilon$, for a certain $\varepsilon>0$.

(3) $W(k+1, x(k+1))-W(k, x(k)) \leq-\alpha_{3}(\|e(k)\|)$ for all $x(k) \in X_{N}(k)$ :

Denoting $J^{o}(k)=V_{N}^{o}(k, x(k), p)$ and

$$
J(k+1)=V_{N}\left(k+1, x(k+1), p, \overline{\mathbf{u}}_{N}(k+1), x_{0}^{a}(k+1), \mathbf{u}_{T}^{a}(k+1)\right)
$$


and taking into account the definition of the proposed feasible solution at $k+1$ we have that

$$
\begin{aligned}
J(k+1)-J^{o}(k)= & -\left\|x(k)-x^{a}(0)\right\|_{Q}^{2}-\left\|u(k)-u^{a}(0)\right\|_{R}^{2} \\
& +\ell\left(k+T, x^{a o}(0 ; k), u^{a o}(0 ; k), p\right) \\
& -\ell\left(k, x^{a o}(0 ; k), u^{a o}(0 ; k), p\right)
\end{aligned}
$$

Since the economic cost function is periodic, and considering the optimality of the solution, we have that

$$
\begin{aligned}
J^{o}(k+1)-J^{o}(k) \leq & -\left\|x(k)-x^{a o}(0 ; k)\right\|_{Q}^{2} \\
& -\left\|u(k)-u^{a o}(0 ; k)\right\|_{R}^{2}
\end{aligned}
$$

From the definition of the Lyapunov function, it can be deduced that

$$
W(k+1, x(k+1))-W(k, x(k)) \leq-\left\|x(k)-x^{a o}(0 ; k)\right\|_{Q}^{2}
$$

This means that the increment of $W(k, x(k))$ is a function of the difference between the state and the artificial state. In virtue of lemma 2 we have that this difference is a measure of the tracking error $e(k)$. That is, there exists a $\mathcal{K}_{\infty}$ function $\alpha_{3}(\cdot)$ such that

$$
\left\|x(k)-x^{a o}(0 ; k)\right\|_{Q}^{2} \geq \alpha_{3}(\|e(k)\|) .
$$

Then we have that

$$
W(k+1, x(k+1))-W(k, x(k)) \leq-\alpha_{3}(\|e(k)\|)
$$

This proves that $W(k, x(k))$ is a Lyapunov function and in virtue of theorem 3 for $\Omega(k)=X_{N}(k)$ and implies that the optimal trajectory is asymptotic stable.

265

\subsubsection{Technical lemmata}

This proof is based in three technical lemmas. The first one is the most important and states that if the system reaches the optimal artificial trajectory, i.e. $x(k)=x^{a o}(0 ; k, p)$, then the artificial trajectory has also reached the optimal

Lemma 1. Let $x(k)$ be such that the solution of the optimization problem $P_{N}(k, x(k), p)$ satisfies $x(k)=x^{a o}(0 ; k)$, then $\overline{\mathbf{x}}_{T}^{o}(k)=\mathbf{x}_{T}^{a o}(k, p)=\mathbf{x}_{T}^{\star}(k)$ and $\overline{\mathbf{u}}_{T}^{o}(k)=$ $\mathbf{u}_{T}^{a o}(k, p)=\mathbf{u}_{T}^{\star}(k)$.

Proof. The proof will be done by contradiction. Assume that $\left(\mathbf{x}_{T}^{a o}, \mathbf{u}_{T}^{a o}\right)$ is not the optimal trajectory $\left(\mathbf{x}_{T}^{\star}(k), \mathbf{u}_{T}^{\star}(k)\right)$, then from the uniqueness of 4 we have that

$$
\sum_{j=0}^{T-1} \ell\left(k+j, x^{a o}(j), u^{a o}(j), p\right)>T L_{\infty}(p)
$$


Let denote the sequences

$$
\left(\hat{\mathbf{x}}_{T}^{a}(k), \hat{\mathbf{u}}_{T}^{a}(k)\right)=\beta\left(\mathbf{x}_{T}^{a o}(k), \mathbf{u}_{T}^{a o}(k)\right)+(1-\beta)\left(\mathbf{x}_{T}^{\star}(k), \mathbf{u}_{T}^{\star}(k)\right)
$$

where $\beta \in(0,1]$.

Since $\left(\mathbf{x}_{T}^{a o}(k), \mathbf{u}_{T}^{a o}(k)\right)$ and $\left(\mathbf{x}_{T}^{\star}(k), \mathbf{u}_{T}^{\star}(k)\right)$ satisfy constraints $(6 \mathrm{e})-(6 \mathrm{~g})$, by convexity $\left(\hat{\mathbf{x}}_{T}^{a}(k), \hat{\mathbf{u}}_{T}^{a}(k)\right)$ also satisfy them.

The controllability assumption 2 ensures that there exists a sequence of control actions

$$
\hat{\mathbf{u}}_{N}(k)=M(k)\left(x(k)-\hat{x}^{a}(k)\right)+\hat{\mathbf{u}}_{N}^{a}(k)
$$

for a suitable matrix $M(k)$, such that reaches $\hat{x}^{a}(k+N)$ in $N$ steps.

Because the optimal trajectory $\mathbf{x}_{\infty}^{\star}(p)$ and $\mathbf{u}_{\infty}^{\star}(p)$ is contained in the relative interior of the constraint set, a $\beta$ arbitrarily close to 1 can be found such that the resulting trajectory satisfies constraints $(6 \mathrm{bb})-(\sqrt{6 \mathrm{~d}})$ and $(\sqrt{6 \mathrm{~h}})$. Therefore the triplet $\left(\hat{\mathbf{u}}_{N}(k), \hat{x}^{a}(0 ; k), \hat{\mathbf{u}}_{T}^{a}(k)\right)$ is a feasible solution of $P_{N}(k, x(k), p)$. The cost associated to this feasible solution is denoted as $\hat{V}_{N}(k)$ and satisfies that

$$
\hat{V}_{N}(k)=\left\|x(k)-x^{\star}(k ; p)\right\|_{H(k)}^{2}+\sum_{j=0}^{T-1} \ell\left(k+j, \hat{x}^{a}(j ; k), \hat{u}^{a}(j ; k), p\right)
$$

for a certain matrix $H(k)$. Taking a constant $\lambda_{H}$ as the maximum eigenvalue of $H(j)$ for all $j \in \mathbb{I}_{[0, T-1]}$ and considering the optimality of the solution we have that

$$
\hat{V}_{N}(k) \leq \lambda_{H}\left\|x(k)-\hat{x}^{a}(0 ; k)\right\|^{2}+\sum_{j=0}^{T-1} \ell\left(k+j, \hat{x}^{a}(j ; k), \hat{u}^{a}(j ; k), p\right)
$$

From the optimality of the solution this cost is such that $\hat{V}_{N}(k) \geq V_{N}^{o}(k ; x(k), p)$. Since the optimal solution at $x(k)=x^{a o}(0 ; k)$ is $\left(\mathbf{u}_{N}^{a o}, x^{a o}(0 ; k), \mathbf{u}_{N}^{a o}\right)$ we have that

$$
V_{N}^{o}(k ; x(k), p)=\sum_{j=0}^{T-1} \ell\left(k+j, x^{a o}(j ; k), u^{a o}(j ; k), p\right)
$$

and then

$$
\hat{V}_{N}(k) \geq \sum_{j=0}^{T-1} \ell\left(k+j, x^{a o}(j ; k), u^{a o}(j ; k), p\right)
$$

On the other hand, from the definition of $\left(\hat{\mathbf{x}}_{T}^{a}(k), \hat{\mathbf{u}}_{T}^{a}(k)\right)$ and taking into account that $x(k)=x^{a o}(0 ; k)$, we have that

$$
x(k)-\hat{x}^{a}(0 ; k)=(1-\beta)\left(x(k)-x^{\star}(k)\right)
$$


Besides, from the convexity of $\ell(k, x, u, p)$ we have that

$$
\begin{aligned}
\sum_{j=0}^{T-1} \ell\left(k+j, \hat{x}^{a}(j ; k), \hat{u}^{a}(j ; k), p\right) \leq & \beta \sum_{j=0}^{T-1} \ell\left(k+j, x^{a o}(j ; k), u^{a o}(j ; k), p\right) \\
& +(1-\beta) T L_{\infty}(p)
\end{aligned}
$$

Using (10) and 12 the following inequality is obtained:

$$
\begin{aligned}
\hat{V}_{N}(k) \leq & (1-\beta)^{2} \lambda_{H}\left\|x(k)-x^{\star}(k)\right\|^{2} \\
& +\beta \sum_{j=0}^{T-1} \ell\left(k+j, x^{a o}(j ; k), u^{a o}(j ; k), p\right) \\
& +(1-\beta) T L_{\infty}(p)
\end{aligned}
$$

The right hand side of this inequality will be denoted as $F(\beta)$. This function is such that

$$
F(1)=\sum_{j=0}^{T-1} \ell\left(k+j, x^{a o}(j ; k), u^{a o}(j ; k), p\right)
$$

and hence, from 111 we have that $F(\beta) \geq F(1)$. Taking the partial of $F(\cdot)$ with respect to $\beta$ function we have that

$$
\begin{aligned}
\frac{\partial F}{\partial \beta}= & -2(1-\beta) \lambda_{H}\left\|x(k)-x^{\star}(k)\right\|^{2} \\
& +\sum_{j=0}^{T-1} \ell\left(k+j, x^{a o}(j ; k), u^{a o}(j ; k), p\right)-T L_{\infty}(p)
\end{aligned}
$$

290

Evaluating this derivative at $\beta=1$

$$
\left.\frac{\partial F}{\partial \beta}\right|_{\beta=1}=\sum_{j=0}^{T-1} \ell\left(k+j, x^{a o}(j ; k), u^{a o}(j ; k), p\right)-T L_{\infty}(p)
$$

From the initial assumption we have that

$$
\left.\frac{\partial F}{\partial \beta}\right|_{\beta=1}>0
$$

Then there exists a $\beta$ arbitrarily close to one such that $F(\beta)<F(1)$. Summarizing we have proved that for a certain $\beta$,

$$
F(1) \leq F(\beta)<F(1)
$$

which is a contradiction and hence, the lemma is proved.

The following two lemmas are necessary to derive the bounds of the Lya295 
Lemma 2. Consider that $x(0) \in X_{N}$, then the optimal solution at each sampling time is such that there exists a $\mathcal{K}_{\infty}$ function $\theta$ such that

$$
\left\|x(k)-x^{a o}(0 ; k)\right\| \geq \theta\left(\left\|x(k)-x^{\star}(k)\right\|\right)
$$

Proof. First we will prove that $\left\|x(k)-x^{a o}(0 ; k)\right\|=0$ iff $\left\|x(k)-x^{\star}(k)\right\|=0$. If $\left\|x(k)-x^{a o}(0 ; k)\right\|=0$, then thanks to the lemma 1, we infer that $\left\|x(k)-x^{\star}(k)\right\|$ is zero.

On the other hand if $\left\|x(k)-x^{\star}(k)\right\|=0$, then the triplet $\left(\mathbf{u}_{N}^{\star}(k), x^{\star}(k), \mathbf{u}_{T}^{\star}(k)\right)$ is the optimal solution to the optimization problem and hence $\left\|x(k)-x^{a o}(0 ; k)\right\|=$ 0 .

As a consequence of this statement, it can be inferred that $\left\|x(k)-x^{a o}(0 ; k)\right\|>$ 0 for all $\left\|x(k)-x^{\star}(k)\right\|>0$.

Finally the optimization problem $P_{N}(k, x, p)$ is a multiparametric convex optimization problem and the optimizers are continuous functions of the state $x$ defined in the compact set $X_{N}\left[25\right.$. Then $x^{a o}(0 ; k)$ is a continuous function of $x(k)$ and hence $x(k)-x^{a o}(0 ; k)$ is a continuous function of $x(k)-x^{\star}(k)$.

From [26] we derive that there exists a $\mathcal{K}_{\infty}$ function such that

$$
\left\|x(k)-x^{a o}(0 ; k)\right\| \geq \theta\left(\left\|x(k)-x^{\star}(k)\right\|\right)
$$

Lemma 3. There exists $a \varepsilon>0$ and a $\mathcal{K}_{\infty}$ function $\alpha_{2}(\cdot)$ such that

$$
W(k, x(k)) \leq \alpha_{2}\left(\left\|x(k)-x^{\star}(k)\right\|\right)
$$

for all $\left\|x(k)-x^{\star}(k)\right\| \leq \varepsilon$.

Proof. The controllability Assumption 2 ensures that there exists a sequence of control actions

$$
\tilde{\mathbf{u}}_{N}(k)=M(k)\left(x(k)-x^{\star}(k ; p)\right)+\mathbf{u}_{N}^{\star}(k ; p)
$$
for a suitable matrix $M(k)$, such that the resulting trajectory reaches $x^{\star}(k+$ $N ; p)$ in $N$ steps.

Furthermore, as the optimal trajectory $\mathbf{x}_{\infty}^{\star}(p)$ and $\mathbf{u}_{\infty}^{\star}(p)$ are contained in the relative interior of the constraint sets, then there exists a small enough positive number $\varepsilon>0$ such that the resulting trajectory $\tilde{\mathbf{x}}_{N}(k)$ is admissible if $\left\|x(k)-x^{\star}(k ; p)\right\| \leq \varepsilon$.

Consequently the triplet $\left(\tilde{\mathbf{u}}_{N}(k), x^{\star}(k), \mathbf{u}_{T}^{\star}(k)\right)$ is a feasible solution of $P_{N}(k, x(k), p)$ and the corresponding cost is

$$
V_{N}\left(k, x(k), p, \tilde{\mathbf{u}}_{N}(k), x^{\star}(k), \mathbf{u}_{T}^{\star}(k)\right)=\left\|x(k)-x^{\star}(k ; p)\right\|_{H(k)}^{2}+T L_{\infty}(k)
$$

for a given matrix $H(k)$. Taking a constant $\lambda_{H}$ as the maximum eigenvalue of $H(j)$ for all $j \in \mathbb{I}_{[0, T-1]}$ and considering the optimality of the solution we have 320 that

$$
V_{N}^{o}(k, x(k), p) \leq \lambda_{H}\left\|x(k)-x^{\star}(k ; p)\right\|^{2}+T L_{\infty}(k)
$$


and then

$$
W(k, x(k)) \leq \lambda_{H}\left\|x(k)-x^{\star}(k ; p)\right\|^{2}
$$

The lemma is proved taking $\alpha_{2}(s)=\lambda_{H} s^{2}$.

Remark 2. Stability is guaranteed for any weight matrices that satisfy the design assumptions. A high weight in the tracking term affects the convergence speed of the real trajectory to the optimal trajectory, however this does not necessarily imply a better average economic performance. However, neither does a low weight that yields a slow convergence. The tuning of this weights define the trajectories during the transient, in a way similar to the weights of a standard MPC for regulation. In the proposed scheme, the performance can be tuned modifying these weights with respect to the economic cost of the artificial trajectory. The tuning has to be done ad-hoc for each application. A design that guarantees a convergence optimal from an economic point of view would have to include the economic cost in the tracking term.

Remark 3. The proposed controller has a number of interesting properties:

(i) The proposed controller guarantees convergence of the closed-loop system to the optimal trajectory $\left(\mathbf{x}_{\infty}^{\star}(p), \mathbf{u}_{\infty}^{\star}(p)\right)$ and the calculation of this trajectory by a DRTO is not required.

(ii) The feasibility region of the optimization problem does not depend on the economic stage cost function. The proposed optimization problem is guaranteed to be recursively feasible even if the economic cost function changes (and hence, the corresponding optimal periodic trajectory). Besides, if the parameter $p$ is varying and converges to a constant value $p_{\infty}$, the control law steers the system to the optimal trajectory $\left(\mathbf{x}_{\infty}^{\star}\left(p_{\infty}\right), \mathbf{u}_{\infty}^{\star}\left(p_{\infty}\right)\right)$.

(iii) The proposed optimization problem is the minimization of a convex function subject to linear constraints that can be efficiently solved using specialized algorithms 4. In fact, for some choices of economic cost functions, such as linear or quadratic functions, the optimization problem is a standard quadratic programming problem.

(iv) The domain of attraction of the proposed controller, $X_{N}$ is in general very large and particularly, larger than the set of initial states that can reach a particular optimal trajectory $\mathbf{x}_{\infty}(p)$ in $N$ steps.

(v) The control law $\kappa_{N}\left(k, x(k), \mathbf{r}_{k}\right)$ is Lipschitz continuous with respect the state $x(k)$. As the model is continuous, then the closed-loop system is input-to-state stable (ISS) with respect to additive disturbances [18, whenever the evolution of the plant is admissible. This implies that the closed-loop system is robust to small variations of the disturbance signal $w(k)$ and/or the matrices $A(k), B(k)$.

(vi) The proposed controller takes into account the economic cost of the transient, since the dynamic real time optimizer is integrated into de predictive controller. The artificial trajectory can be seen as a reachable periodic trajectory that minimizes the average cost function. This trajectory is updated at each sample time and converges to the optimal trajectory. 


\section{Application to a four tank plant}

In this section we present the application of the proposed controller to the multivariable laboratory plant of four interconnected tanks with nonlinear dynamics and subject to operational state and input constraints presented in 1, see figure 1. The inputs are the water flows through the two pumps denoted $q_{a}, q_{b}$. The outputs are the water levels in the lower two tanks $\left(h_{1}\right.$ and $\left.h_{2}\right)$ and the states of the model are the water levels of the four tanks $\left(h_{1}, h_{2}, h_{3}\right.$ and $\left.h_{4}\right)$.

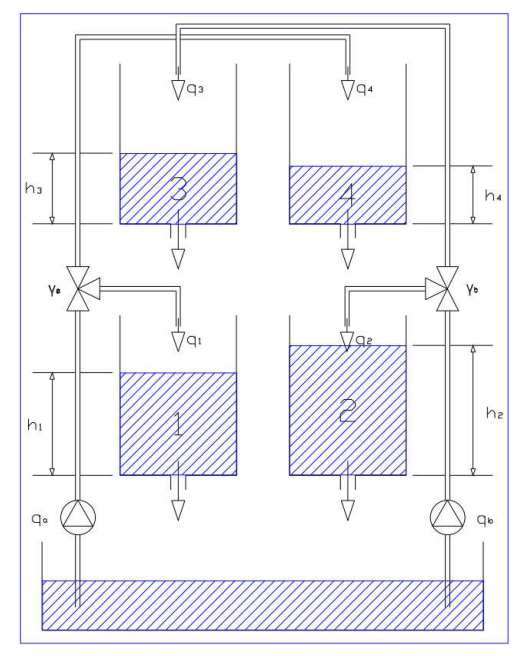

Figure 1: Four Tank scheme.

The four tank plant can be approximated by the following nonlinear model [16]:

$$
\begin{aligned}
& \dot{h}_{1}=-\frac{a_{1}}{A} \sqrt{2 g h_{1}}+\frac{a_{3}}{A} \sqrt{2 g h_{3}}+\frac{\gamma_{1}}{3600 \cdot A} q_{a} \\
& \dot{h}_{2}=-\frac{a_{2}}{A} \sqrt{2 g h_{2}}+\frac{a_{4}}{A} \sqrt{2 g h_{4}}+\frac{\gamma_{2}}{3600 \cdot A} q_{b} \\
& \dot{h}_{3}=-\frac{a_{3}}{A} \sqrt{2 g h_{3}}+\frac{1-\gamma_{2}}{3600 \cdot A} q_{a} \\
& \dot{h}_{4}=-\frac{a_{4}}{A} \sqrt{2 g h_{4}}+\frac{1-\gamma_{1}}{3600 \cdot A} q_{b}
\end{aligned}
$$

subject to the following state and input constraints

$$
\begin{aligned}
Q_{j}^{\text {min }} \leq q_{j} \leq Q_{j}^{\max } & j=a, b \\
H_{i}^{\text {min }} \leq h_{i} \leq H_{i}^{\text {max }} & i=1,2,3,4
\end{aligned}
$$

where the values of the parameters of the system can be found in table 1 . The nonlinear model will be used to carry out all the simulations of this section.

To apply the proposed controller, we will use a discrete time linear model obtained by linearizing the nonlinear model around the equilibrium point defined 


\begin{tabular}{|c|c|c|c|}
\hline & Value & Unit & Description \\
\hline$H_{1}^{\max }$ & 1.20 & $\mathrm{~m}$ & Maximum level of the tank 1 \\
\hline$H_{2}^{\max }$ & 1.20 & $\mathrm{~m}$ & Maximum level of the tank 2 \\
\hline$H_{3}^{\max }$ & 1.20 & $\mathrm{~m}$ & Maximum level of the tank 3 \\
\hline$\dot{H_{4}^{\max }}$ & 1.20 & $\mathrm{~m}$ & Maximum level of the tank 4 \\
\hline$H_{i}^{\min }$ & 0.2 & $\mathrm{~m}$ & Minimum level of the tanks \\
\hline$Q_{a}^{\max }$ & 2.50 & $m^{3} / h$ & Maximal flow \\
\hline$Q_{b}^{\max }$ & 2.50 & $m^{3} / h$ & Maximal flow \\
\hline$Q_{i}^{\min }$ & 0 & $m^{3} / h$ & Minimal flow \\
\hline$a_{1}$ & $1.341 e^{-4}$ & $m^{2}$ & Discharge constant of the tank 1 \\
\hline$a_{2}$ & $1.533 e^{-4}$ & $m^{2}$ & Discharge constant of the tank 2 \\
\hline$a_{3}$ & $9.322 e^{-5}$ & $m^{2}$ & Discharge constant of the tank 3 \\
\hline$a_{4}$ & $9.061 e^{-5}$ & $m^{2}$ & Discharge constant of the tank 4 \\
\hline$A$ & 0.03 & $m^{2}$ & Cross-section of all tanks \\
\hline$\gamma_{a}$ & 0.3 & n.u. & Parameter of the 3 -ways valve \\
\hline$\gamma_{b}$ & 0.4 & n.u. & Parameter of the 3 -ways valve \\
\hline$h_{1}^{o}$ & 0.627 & $\mathrm{~m}$ & Equilibrium level tank 1 \\
\hline$h_{2}^{o}$ & 0.636 & $\mathrm{~m}$ & Equilibrium level tank 2 \\
\hline$h_{3}^{o}$ & 0.652 & $\mathrm{~m}$ & Equilibrium level tank 3 \\
\hline$h_{4}^{o}$ & 0.633 & $\mathrm{~m}$ & Equilibrium level tank 4 \\
\hline$Q_{a}^{o}$ & 1.6429 & $m^{3} / h$ & Equilibrium flow a \\
\hline$Q_{b}^{o}$ & 2.0000 & $m^{3} / h$ & Equilibrium flow b \\
\hline
\end{tabular}

Table 1: Parameters of the four tank plant

by $h_{i}^{o}, q_{j}^{o}$ with $i=1,2,3,4$ and $j=a, b$, (see table 1 ). To reduce the steady linearization errors, the state of the linear model is defined as $x_{i}=\sqrt{h_{i}}-$ $\sqrt{h_{i}^{o}}$ and inputs are defined as $u_{j}=q_{j}-q_{j}^{o}$ where $j=a, b$ and $i=1,2,3,4$. The linearized model has been discretized using the Tusting method with a sampling time of five seconds. The resulting matrices for the linear model are the following:

$$
A=\left(\begin{array}{cccc}
0.9419 & 0 & 0.0401 & 0 \\
0 & 0.9334 & 0 & 0.0380 \\
0 & 0 & 0.9587 & 0 \\
0 & 0 & 0 & 0.9607
\end{array}\right) ; \quad B=\left(\begin{array}{cc}
0.0083 & 0.0003 \\
0.0004 & 0.0111 \\
0 & 0.0168 \\
0.0196 & 0
\end{array}\right) ;
$$

Note that in this application, the model is time invariant and disturbances are 375 not taken into account.

The economic cost function $\ell(k, x, u, p)$ is made of two terms. The first term penalizes the water flow through the pumps, while the second term is inversely proportional to the water stored in the lower tanks.

$$
\ell(k, x, u, p)=\left(q_{a}(u)^{2}+c(k) q_{b}(u)^{2}\right)+p \frac{V_{\min }}{A\left(h_{1}(x)+h_{2}(x)\right)}
$$

where $V_{\min }$ is the minimum volume of water that can be stored in the lower 
two tanks, that is

$$
V_{\min }=A \cdot\left(H_{1}^{\min }+H_{2}^{\text {min }}\right)
$$

The parameter $c(k)$ is the unitary cost of the cost of the flow $q_{b}$. This is time varying and its evolution is defined by the following periodic function with period 150 seconds:

$$
c(k)=0.15 \sin \left(\frac{2 \cdot \pi \cdot t}{30}\right)+1 \quad t=0, \cdots, N_{r}-1
$$

The parameter $p$ can change abruptly during operation, modeling a different economic criterion. In this case study, we consider two scenarios: In the first scenario, $p$ is constant and equal to 15 and then there is an abrupt change at time step $t=300 \mathrm{~s}$ in which $p$ changes from 15 to 25 leading to a second scenario.

Remark 4. Variable $p$ is introduced to denote a sudden change in the economic criterion. The key issue is that the EMPC considers that $p$ is constant, so when 390 a sudden change occurs, if the controller is not defined appropriately and the problems constraints depend of the economic cost function, feasibility issues may arise. To simplify the example, $p$ has been given a numeric value that changes abruptly. Note that no assumptions are made on the class of functions or the effect of $p$, so in general, the economic costs may be completely different.

The cost matrices $Q, R$ of the proposed control scheme for both scenarios are the following

$$
Q=\mathbb{I}^{4}, \quad R=\mathbb{I}^{2}
$$

The resulting optimization problem has been solved using the fmincon function of Matlab 2013a. The number of decision variables of the problem is 120 .

The model used to carry out the simulations is the nonlinear model $16 \mathrm{a}-16 \mathrm{~d}$ The initial state of all the simulations is

$$
\left(\begin{array}{l}
h_{1}(0) \\
h_{2}(0) \\
h_{3}(0) \\
h_{4}(0)
\end{array}\right)=\left(\begin{array}{l}
0.4594 \\
0.9534 \\
0.4587 \\
0.9521
\end{array}\right)
$$

Figures 2,5 show the closed-loop (blue), artificial (cyan) and optimal economic (red) trajectories of the level of the four tanks for scenario 1. The artificial trajectory level is obtained at each sampling time from the corresponding optimal initial state of the artificial trajectory.

Figures 6 6 show the closed-loop (blue), artificial (cyan) and optimal economic (red) trajectories of the water flows of pumps a and b. The artificial 405 trajectory water flow is obtained at each sampling time from the corresponding optimal initial input of the artificial trajectory. 


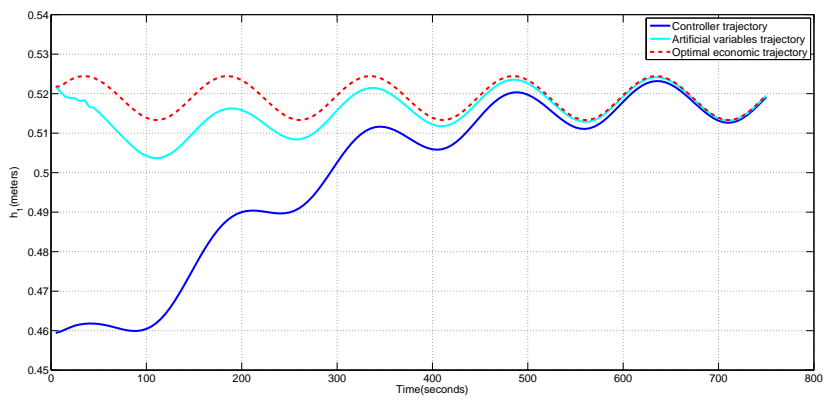

Figure 2: Closed-loop (blue), artificial (cyan) and optimal economic (red) trajectories of the level of tank 1 for scenario 1 .

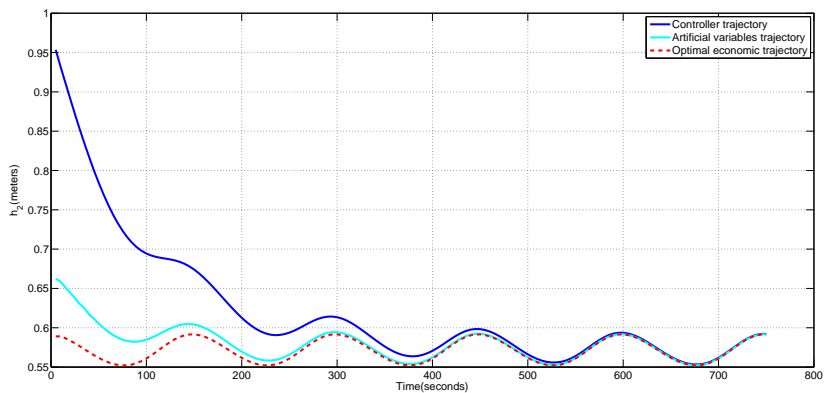

Figure 3: Closed-loop (blue), artificial (cyan) and optimal economic (red) trajectories of the level of tank 2 for scenario 1 .

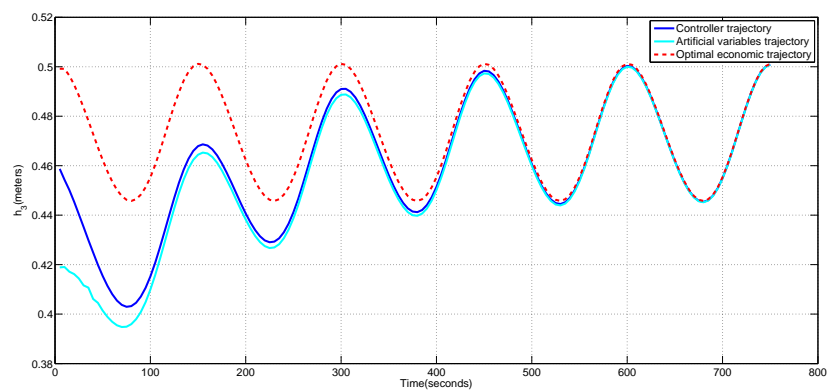

Figure 4: Closed-loop (blue), artificial (cyan) and optimal economic (red) trajectories of the level of tank 3 for scenario 1. 


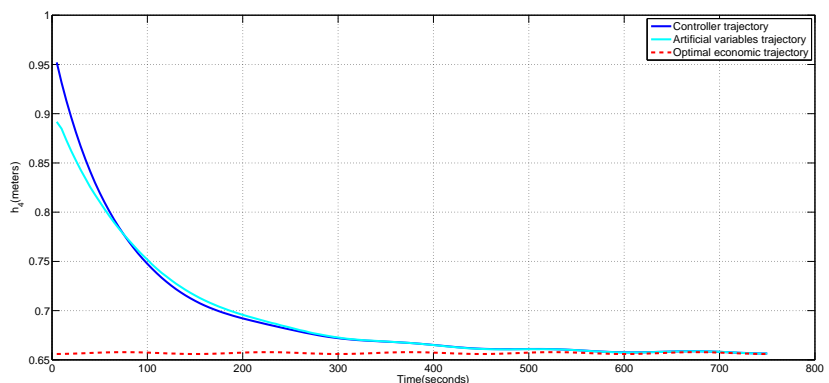

Figure 5: Closed-loop (blue), artificial (cyan) and optimal economic (red) trajectories of the level of tank 4 for scenario 1 .

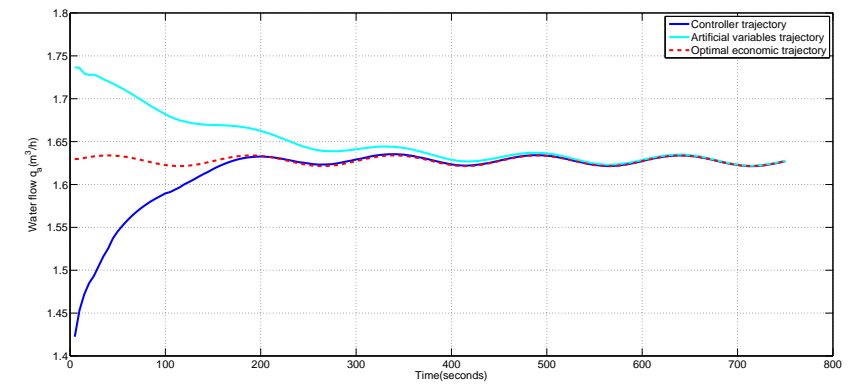

Figure 6: Closed-loop (blue), artificial (cyan) and optimal economic (red) trajectories of the water flow $a$ for scenario 1 .

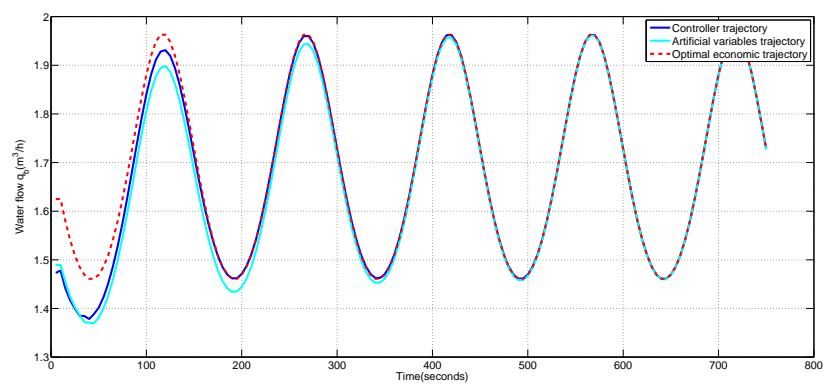

Figure 7: Closed-loop (blue), artificial (cyan) and optimal economic (red) trajectories of the water flow $b$ for scenario 1 . 


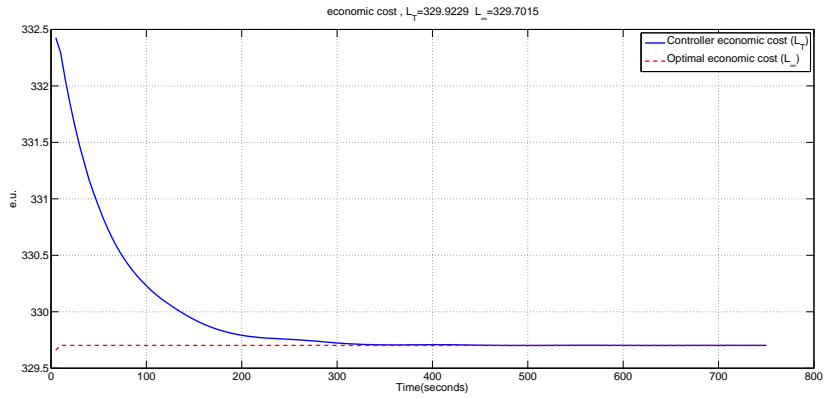

Figure 8: Evolution $\frac{1}{T} \mathcal{L}_{T}(k, \mathbf{x}, \mathbf{u}, \mathbf{p})$.

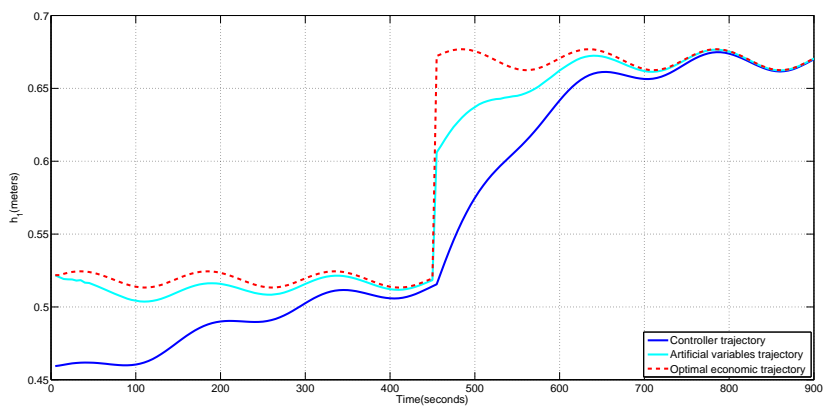

Figure 9: Closed-loop (blue), artificial (cyan) and optimal economic (red) trajectories of the level of tank 1 for scenario 2 .

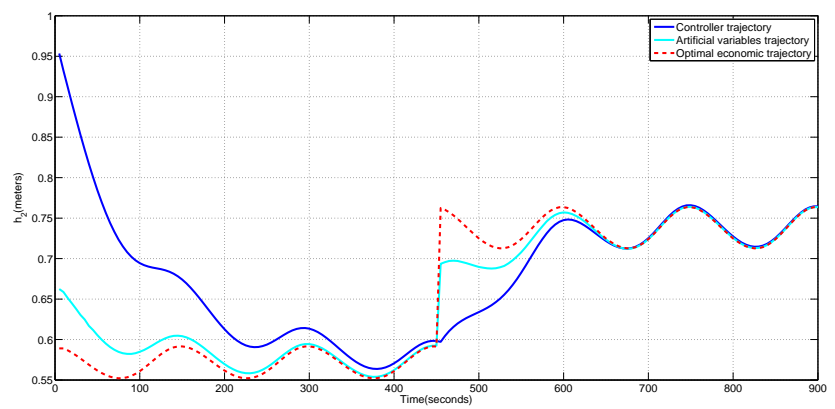

Figure 10: Closed-loop (blue), artificial (cyan) and optimal economic (red) trajectories of the level of tank 2 for scenario 2. 


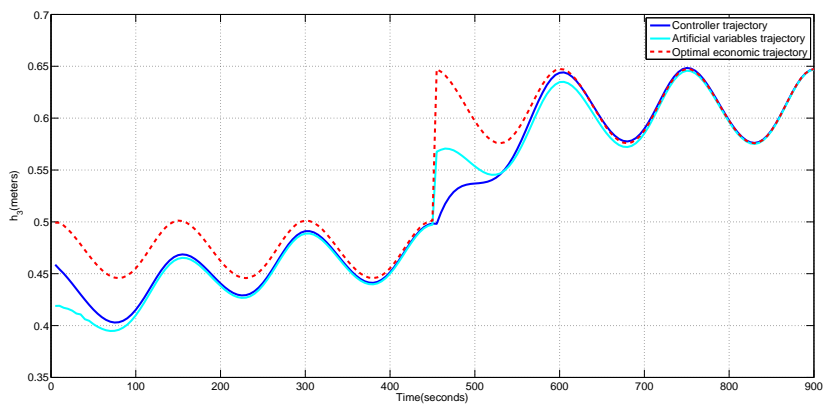

Figure 11: Closed-loop (blue), artificial (cyan) and optimal economic (red) trajectories of the level of tank 3 for scenario 2 .

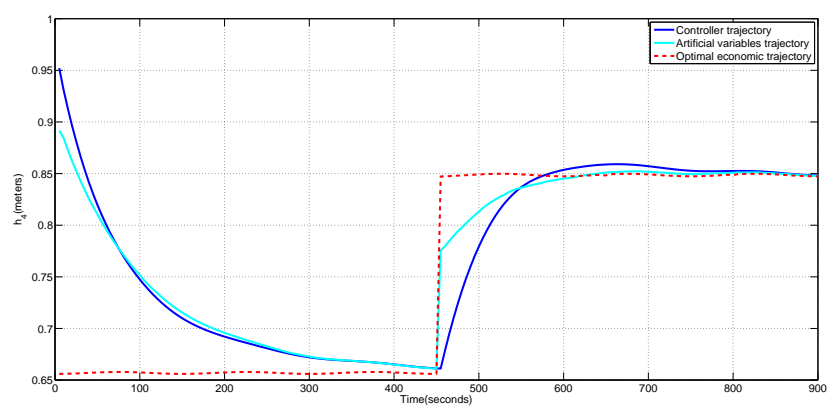

Figure 12: Closed-loop (blue), artificial (cyan) and optimal economic (red) trajectories of the level of tank 4 for scenario 2 .

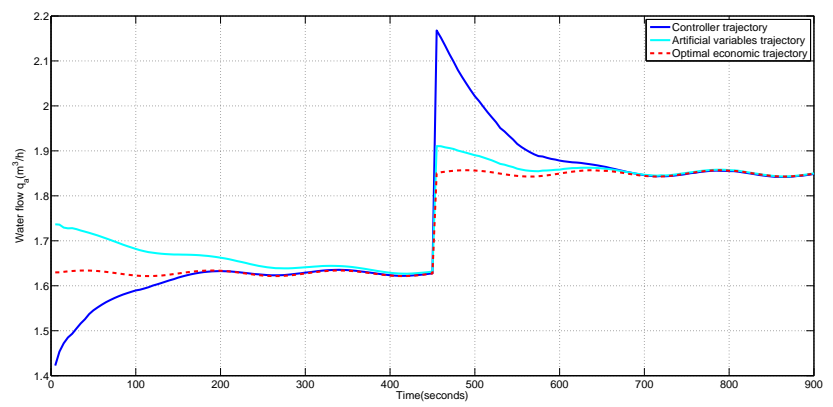

Figure 13: Closed-loop (blue), artificial (cyan) and optimal economic (red) trajectories of the water flow $a$ for scenario 1 . 


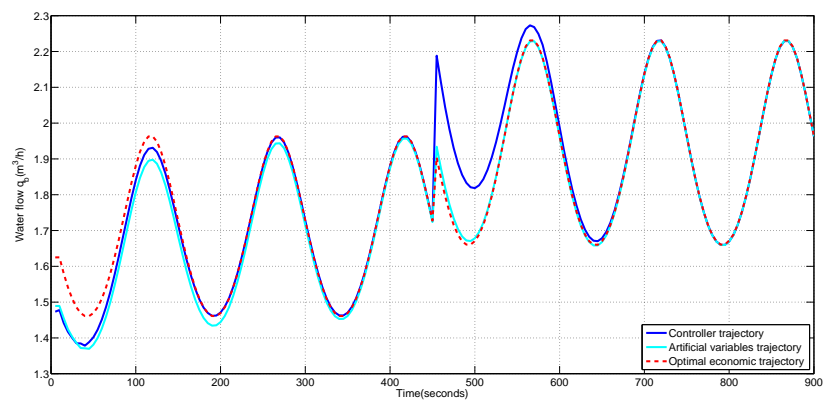

Figure 14: Closed-loop (blue), artificial (cyan) and optimal economic (red) trajectories of the water flow $b$ for scenario 2 .

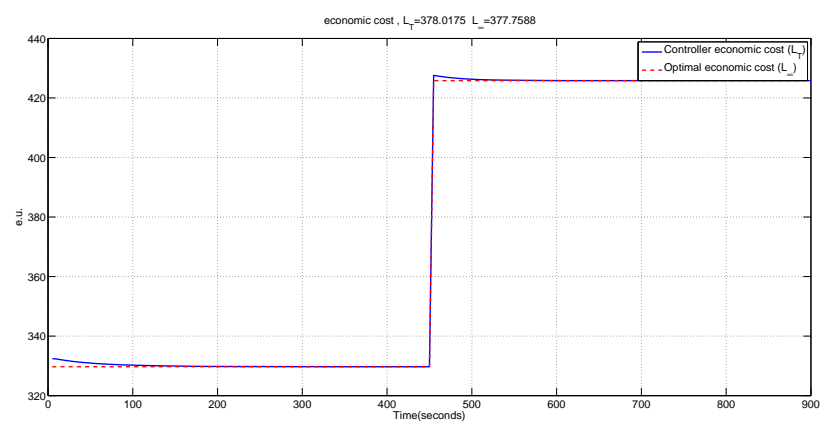

Figure 15: Evolution of $\frac{1}{T} \mathcal{L}_{T}(\mathbf{k}, \mathbf{x}, \mathbf{u}, \mathbf{p})$. 
The simulation starts in an initial state far away from the optimal trajectory, but the system is driven to an appropriate optimal periodic trajectory that minimizes the economic periodic function, i.e., the optimal trajectory. In figure ${ }_{410} 8$ the convergence of $\frac{1}{T} \mathcal{L}_{T}(k, \mathbf{x}, \mathbf{u}, \mathbf{p})$ to $L_{\infty}(k, \mathbf{x}, \mathbf{u}, \mathbf{p})$ is shown.

This implies that the average economic cost in a period of the obtained trajectory converges to cost corresponding to the optimal trajectory.

In the second scenario, the robustness of the proposed controller with respect to abrupt changes in the economic stage cost is shown. In this case, the 415 parameter $p$ changes at $t=300 s$ from 15 to 25 .

Figures 914 show the level and water flow trajectories for scenario 2. The simulations show an abrupt change in all the trajectories at the sampling time in which the economic stage cost is changed. It is important to remark that the corresponding optimal periodic trajectories are very different between the two cost functions considered, which in general implies that the target trajectories for the controller are very different, however, the controller remains feasible because the optimization problem constraints do not depend on the optimal trajectory.

Figure 15 shows the strong change in the optimal average economic cost ${ }_{425}$ when the variable $p$ changes its value.

\section{Application to a drinking water network}

In this example we consider a simplified model of a section of Barcelona's drinking water network (DWN) presented in 21. Figure 16 and table 2 show a scheme of this network which consists on three water tanks, three valves and 430 three water pumps. The network is connected to four demand points from which water is consumed (drinking water demand), and two water supply points from which the water is obtained.

The proposed system is modeled as discrete time invariant model with a sampling time of one hour.

$$
\begin{aligned}
x(k+1) & =A \cdot x(k)+B \cdot u(k)+B_{d} \cdot d(k) \\
0 & =E \cdot u(k)+E_{d} \cdot d(k)
\end{aligned}
$$

${ }_{435}$ where $x(k) \in \mathbb{R}^{3}$ denotes de vector of volume in storage tanks (in $m^{3}$ ) and $u(k) \in \mathbb{R}^{6}$ denotes de vector of water flows through the six actuators given in $\frac{m^{3}}{s}$. Vector $d(k) \in \mathbb{R}^{4}$ denotes the network demands and it is also given in $m^{3}$. The relationship between these variables and the variables of the real model can be found in table 2. Constraint $19 \mathrm{~b}$ models that the DWN must satisfy

440 the water demand defined by $d(k)$ at nodes $N O P 18$ and $N O P 25 B$ as shown in figure 16. The equations that must be fulfilled on the nodes $N O P 18$ and $N O P 25 B$ are the following:

$$
\begin{aligned}
& u_{1}(k)=u_{2}(k)+u_{3}(k)+u_{6}(k) \\
& u_{2}(k)=u_{5}(k)+d_{2}(k)
\end{aligned}
$$




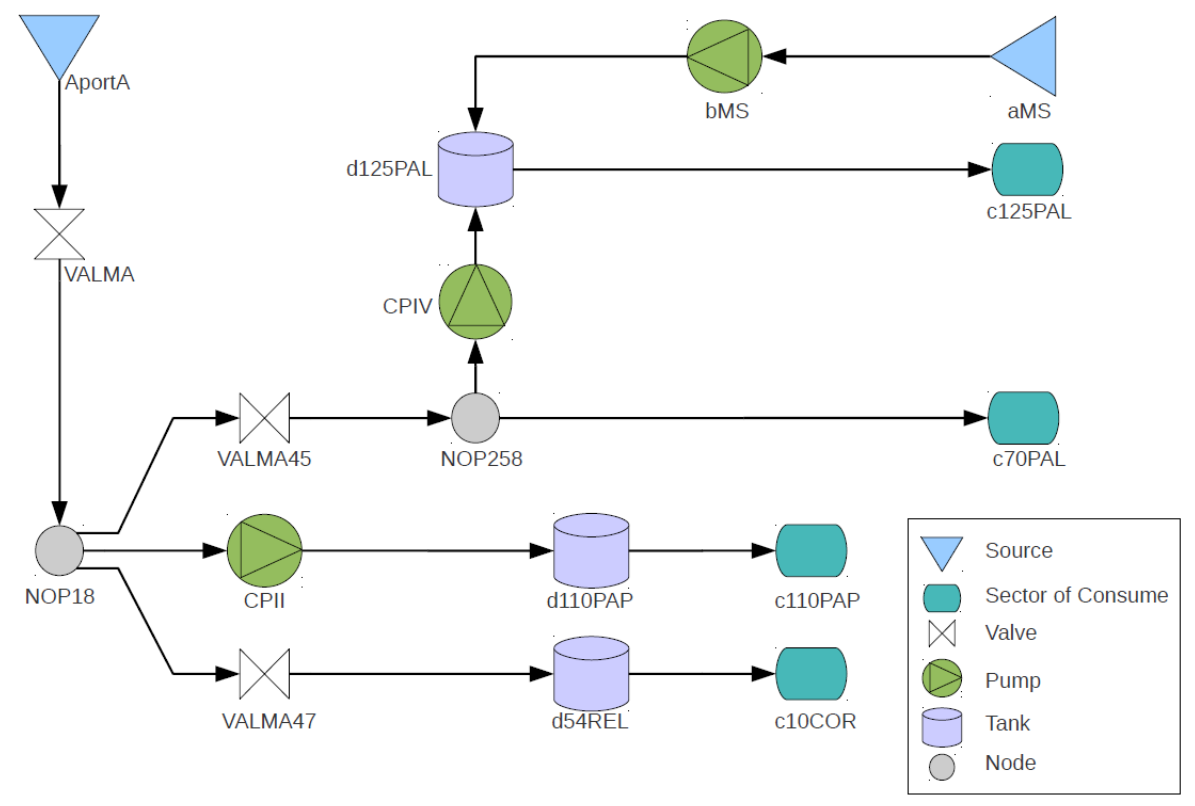

Figure 16: Topology of the Three-tanks DWN example

\begin{tabular}{lrr}
\hline Variable & Designed by & Description \\
\hline \hline$u_{1}$ & VALMA & Valve \\
$u_{2}$ & VALMA45 & Valve \\
$u_{3}$ & VALMA47 & Valve \\
$u_{4}$ & bMs & Pump \\
$u_{5}$ & CPIV & Pump \\
$u_{6}$ & CPII & Pump \\
$x_{1}$ & d125PAL & Tank 1 \\
$x_{2}$ & d110PAP & Tank 2 \\
$x_{3}$ & d54REL & Tank 3 \\
$d_{1}$ & c125PAL & Demand point 1 \\
$d_{2}$ & c70PAL & Demand point 2 \\
$d_{3}$ & c101PAP & Demand point 3 \\
$d_{4}$ & c10COR & Demand point 4 \\
$a_{1}$ & AportA & Water supply A \\
$a_{2}$ & aMS & Water supply B \\
\hline \hline
\end{tabular}

Table 2: Glossary of the topology of the DWN example

The matrices $A, B$ and $B_{d}$ that define the model are the following

$$
\begin{gathered}
A=\left(\begin{array}{ccc}
1 & 0 & 0 \\
0 & 1 & 0 \\
0 & 0 & 1
\end{array}\right) ; \\
B=\left(\begin{array}{cccccc}
0 & 0 & 0 & 3600 & 3600 & 0 \\
0 & 0 & 0 & 0 & 0 & 3600 \\
0 & 0 & 3600 & 0 & 0 & 0
\end{array}\right) ; \\
B_{d}=\left(\begin{array}{cccc}
-3600 & 0 & 0 & 0 \\
0 & 250 & -3600 & 0 \\
0 & 0 & 0 & -3600
\end{array}\right) ;
\end{gathered}
$$


The matrices $E, E_{d}$ are the following

$$
\begin{aligned}
E & =\left(\begin{array}{cccccc}
1 & -1 & -1 & 0 & 0 & -1 \\
0 & 1 & 0 & 0 & -1 & 0
\end{array}\right) ; \\
E_{d} & =\left(\begin{array}{cccc}
0 & 0 & 0 & 0 \\
0 & -1 & 0 & 0
\end{array}\right) ;
\end{aligned}
$$

System $19 \mathrm{a}$ is subject to the following constraints on inputs and state

$$
\begin{aligned}
& U_{\min } \leq u(k) \leq U_{\max } \\
& X_{\min } \leq x(k) \leq X_{\max }
\end{aligned}
$$

where

$$
\begin{aligned}
U_{\text {min }} & =\left(\begin{array}{llllll}
0 & 0 & 0 & 0 & 0 & 0
\end{array}\right)^{T} \\
U_{\max } & =\left(\begin{array}{llllll}
1.2970 & 0.0500 & 0.1200 & 0.0150 & 0.0317 & 0.0220
\end{array}\right)^{T} \\
X_{\min } & =\left(\begin{array}{lll}
0 & 0 & 0
\end{array}\right)^{T} \\
X_{\max } & =\left(\begin{array}{lll}
470 & 960 & 3100
\end{array}\right)^{T}
\end{aligned}
$$

The economic goal of the management of the DWN is to minimize the cost of water production and water transport supplying a periodic demand, typically of $24 \mathrm{~h}$. There are two operational goals that are included in the economic cost function. The operational goals are to guarantee the availability of water in every tank in order to satisfy the stochastic changes on the periodic demands and operate the network under smooth control actions.

The first operational criteria is taken into account including a penalty equal to the square of the deviation of the volume in each tank below the minimum values estimated for robust operation:

$$
J_{S}(k)=\sum_{i=1}^{3}\left\|\delta_{i}(k)\right\|^{2} .
$$

where $\delta_{i}(k)$ denotes the deviation of the stored volume in tank $i$ below the desired minimum volume $x_{i}^{s f}(k)$.

$$
\delta_{i}(k) \triangleq\left\{\begin{array}{cll}
0 & x_{i}(k)>x_{i}^{s f}(k) & i=1,2,3 \\
x_{i}^{s f}(k)-x_{i}(k) & x_{i}(k)<x_{i}^{s f}(k) & i=1,2,3
\end{array}\right.
$$

The second operational criteria is taken into account including a penalty on the square of the variations of the water flows in the pump and valves,

$$
J_{\Delta u}(k) \triangleq\|\Delta u(k)\|^{2}
$$

${ }_{455}$ where $\Delta u(k)=u(k)-u(k-1)$. 


\begin{tabular}{cccccccc}
\hline$\alpha$ & $u_{1}$ & $u_{2}$ & $u_{3}$ & $u_{4}$ & $u_{5}$ & $u_{6}$ & time zone \\
\hline \hline$\alpha_{1}$ & 2 & 0 & 0 & 2 & 0 & 0 & $0-24$ hours \\
$\alpha_{2}(k)$ & 0 & 0 & 0 & 0.5 & 0.1 & 1 & $0-9$ hours \\
$\alpha_{2}(k)$ & 0 & 0 & 0 & 3 & 4 & 3 & $9-20$ hours \\
$\alpha_{2}(k)$ & 0 & 0 & 0 & 0.1 & 0.5 & 0.5 & $20-24$ hours \\
\hline \hline
\end{tabular}

Table 3: Cost selection

Finally the economic cost of the water is included. This cost takes into account both the cost from the supply points and the transportation cost of operating the valves and pumps of the network.

$$
J_{E}(k, p) \triangleq\left(\alpha_{1}+p \cdot \alpha_{2}(k)\right)^{T} \cdot u(k)
$$

The cost is made up of a fixed water production cost denoted as $\alpha_{1} \in \mathbb{R}^{6}$ and a time-varying (periodic) water cost denoted as $\alpha_{2}(k) \in \mathbb{R}^{6}$. Table 3 shows the value of the cost parameters $\alpha_{1}$ and $\alpha_{2}(k)$ for each water flow. The parameter $p$ is used to express an incremental change in electric prices at certain instant.

Summing up, the economic stage cost function is the following

$$
\ell(k, x, u, p) \triangleq \lambda_{1} \cdot J_{E}(k, p)+\lambda_{2} \cdot J_{S}(k)+\lambda_{3} \cdot J_{\Delta u}(k)
$$

where $\lambda_{1}, \lambda_{2}, \lambda_{3}$ are tuning parameters that set the influence of each objective on the overall economic cost function. The value of the parameters in this example are $\lambda_{1}=100, \lambda_{2}=10, \lambda_{3}=0.005$.

For the tracking term, the weighting matrices are $Q=0.01 * \mathbb{I}^{3}$ and $R=$ $10 * \mathbb{I}^{6}$. The prediction horizon and the periodic horizon are 24 hours and the sampling time is one hour. The initial state of the network is $x(0)=$ $(160.44,646.23,633.89)$ given in $m^{3}$ and $u(0)=u(-1)=(0,0,0,0,0,0)$ given in $m^{3} / h$. The demands on points $c 125 P A L, c 70 P A L, c 110 P A P$ and $c 10 C O R$ are periodic signals with a period of 24 hours, see figure 17

470 In this example, the simulation horizon is six days. We consider a scenario in which the cost changes abruptly after $72 h$ of operation. During the first $72 h$, the parameter $p$ is equal to zero and then the parameter takes the value of $p=1$. This is equivalent to increase the unitary cost of pumping according to a given pricing policy.

${ }_{475}$ For the simulation test, the optimization problem has been implemented using an epigraph formulation [21] and this has been carried out using qpOASES toolbox on Matlab 2013a and an Intel Core $i 7-4700$ with 16 GB of RAM.

Figure 18 shows the evolution of the tree tanks over 144 hours. It can be seen 480 that during the first 72 hours, the optimal volumes of the three tanks is equal to the minimum levels because the costs of the water and transport are constant along the 24 hour period, so the optimal policy is to satisfy the demand and have the minimum amount of water in the tanks. When the cost of the water 


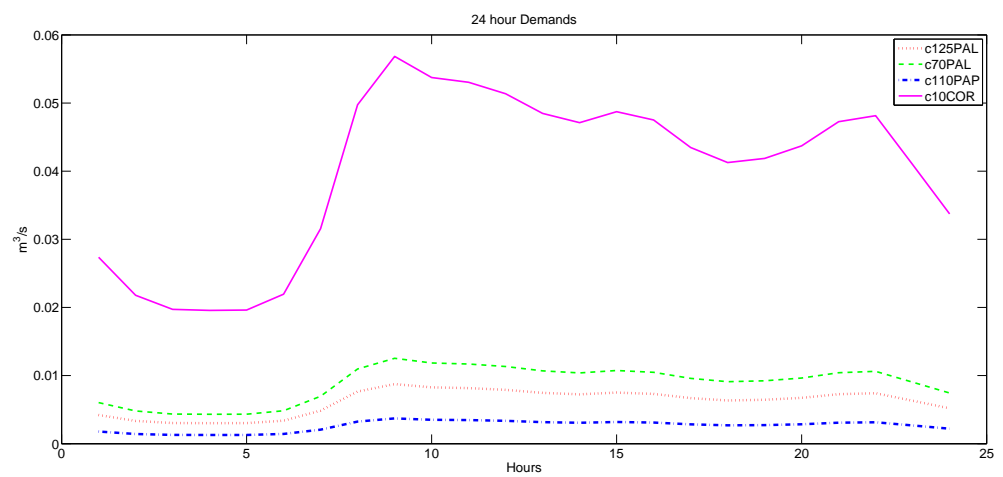

Figure 17: 24h demand on points $c 125 P A L, c 70 P A L, c 110 P A P$ and $c 10 C O R$.
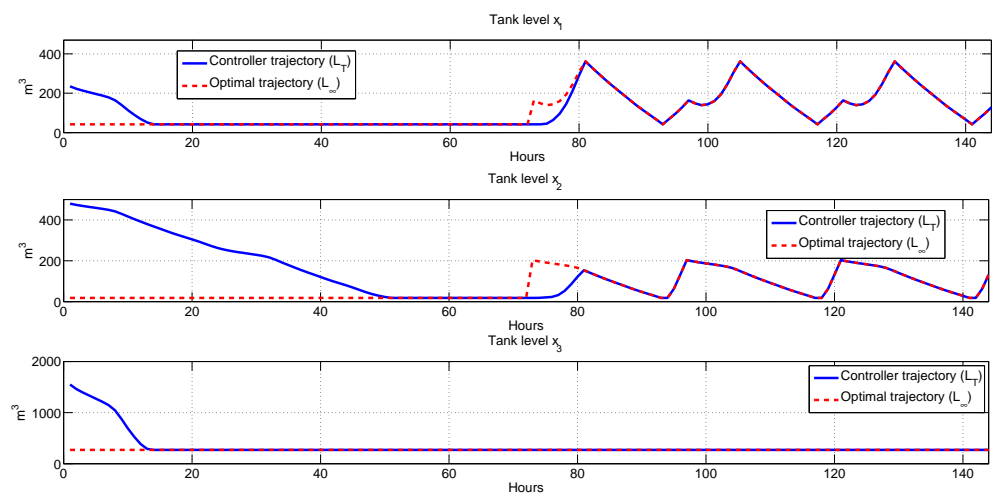

Figure 18: Stored volume in the Tank $\left[\mathrm{m}^{3}\right]$.

is modified and becomes time varying, then it is more profitable to accumulate water when the cost is lower, to provide this water to the demand during the high cost time periods. In addition, it can be seen, that the supply points are also used at different time periods, depending on the value of their corresponding $\alpha_{2}(k)$.

Figures 19 24 show the corresponding trajectories of optimal periodic control inputs (red) $u^{\star}(k)$ and closed-loop (blue) inputs $u(k)$. Notice that during the first 72 hours, no water is accumulated, and in fact, the initial stored volume of water is used to satisfy the demand. After parameter $p$ is changed, the new pricing policy produces an significant change in the optimal operation of the plant. This can be seen in figures 24 24 where the flows of the pumps change from an smooth periodic operation to a fast transition between its limits. This is also illustrated in figure 18, where the evolution of the volume of water in the tanks 1 and 2 changes from an steady evolution to a periodic evolution, storing 


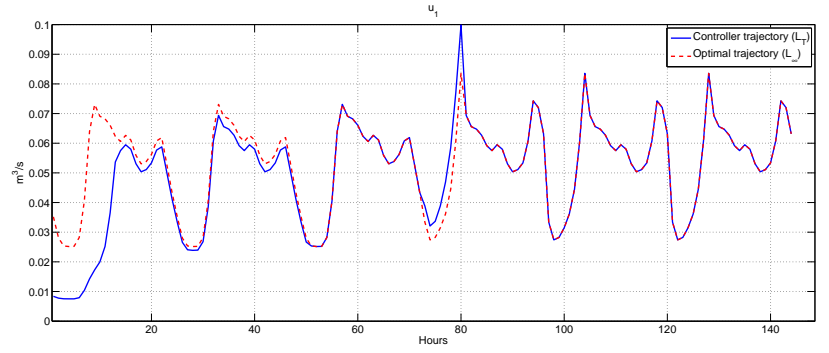

Figure 19: Optimal periodic (red) and closed-loop (blue) trajectories of valve $V A L M A$.

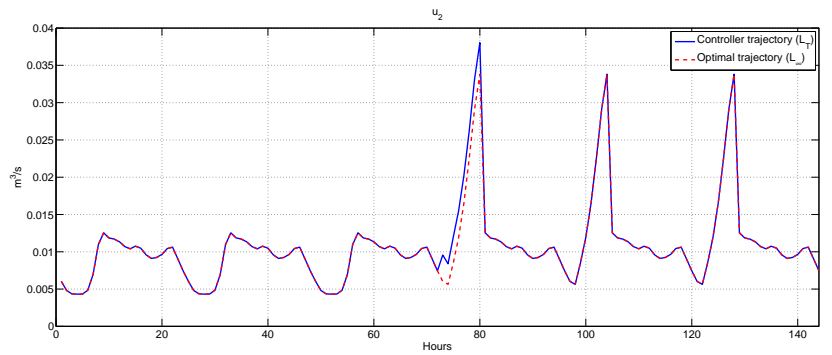

Figure 20: Optimal periodic (red) and closed-loop (blue) trajectories of valve VALMA45.

water whenever the pumping price is lower.

Figure 25 shows the average economic cost of the optimal trajectory during a period (red) and optimal cost of the controller optimization problem (blue) trajectories. It can be seen that the optimal cost of the controller converges to the optimal cost as stated in the main theorem. The feasibility and robustness properties of this controller can be observed when the cost changes after $72 \mathrm{~h}$. There is a sudden change in the optimal periodic trajectory when the parameter $p$ changes. Note that the controller continues without loss of feasibility and that the closed-loop trajectories converge to the new optimal ones.

\section{Conclusions}

In this paper, we focus on optimal (non-steady) periodic operation of process systems based on an economic criteria which can change arbitrarily. We have proposed an economic model predictive controller that decides solving a single optimization problem the optimal input to guarantee that the closed-loop system trajectory converges to the optimal periodic trajectory that minimizes the average economic operation cost without needing an a priori calculation of this trajectory by a dynamic real time optimizer. The economic criteria, and hence 515 the corresponding optimal periodic trajectory, can change arbitrarily, without needing to reconfigure the proposed controller while maintaining feasibility. The proposed controller has been applied to a four tank system and to a simplified 


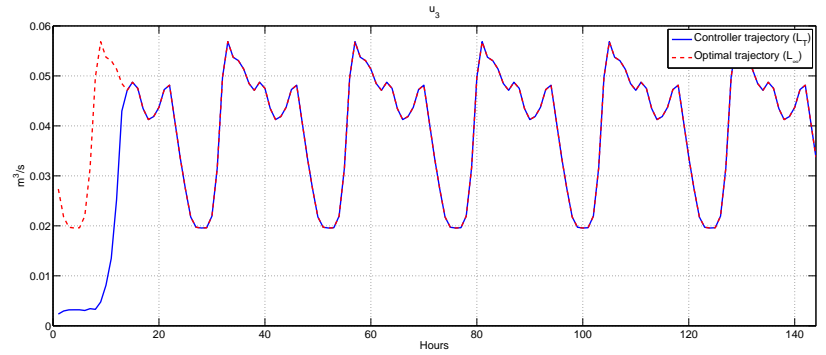

Figure 21: Optimal periodic (red) and closed-loop (blue) trajectories of valve $V A L M A 47$.

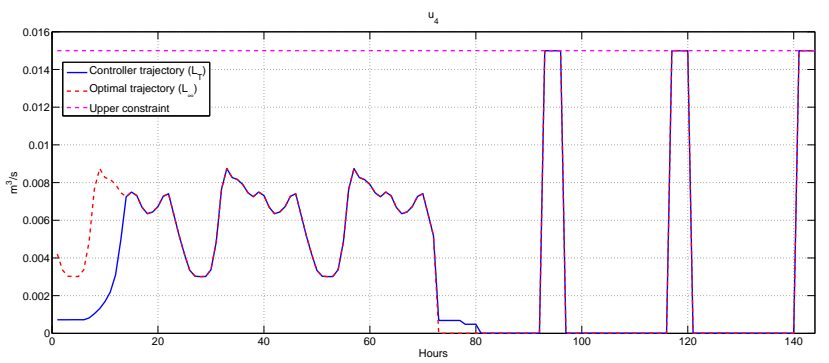

Figure 22: Optimal periodic (red) and closed-loop (blue) trajectories of pump $b M S$.

model of a section of Barcelona's drinking water network, a system in which the operation is intrinsically periodic.

\section{References}

[1] Alvarado, I., 2007. Model predictive control for tracking constrained linear systems. Ph.D. thesis, Univ. de Sevilla.

[2] Angeli, D., Amrit, R., Rawlings, J., 2012. On average performance and stability of economic model predictive control. IEEE Transactions on Automatic Control 57, 816-820.

[3] Bittanti, S., Colaneri, P., 2009. Periodic Systems: Filtering and Control. Springer.

[4] Boyd, S., Vandenberghe, L., 2006. Convex Optimization. Cambridge University Press.

[5] Camacho, E. F., Bordons, C., 2004. Model Predictive Control, 2nd Edition. Springer-Verlag.

[6] Darby, M., Nikolaou, M., Jones, J., Nicholson, D., 2011. Rto: An overview and assessment of current practice. Journal of Process Control 21, $874-$ 884. 


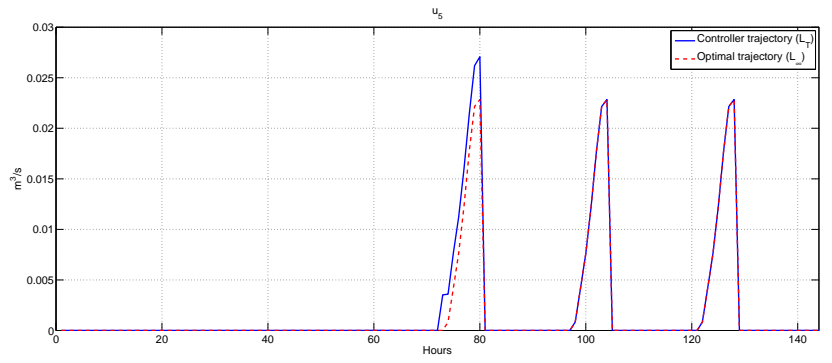

Figure 23: Optimal periodic (red) and closed-loop (blue) trajectories of pump CPIV.

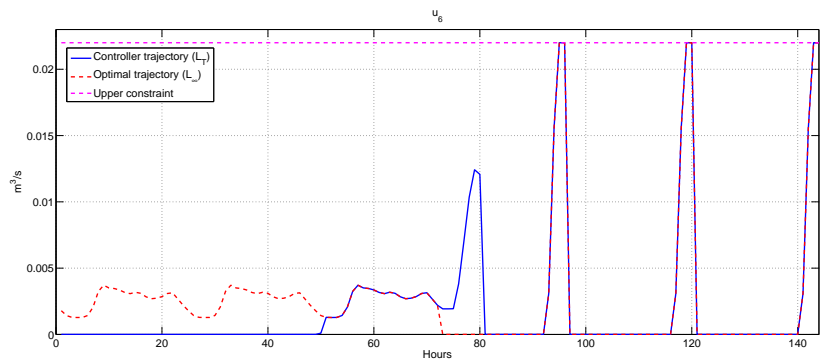

Figure 24: Optimal periodic (red) and closed-loop (blue) trajectories of pump CPII.

[13] Huang, R., Biegler, L., Harinath, E., 2012. Lyapunov stability of econom- 


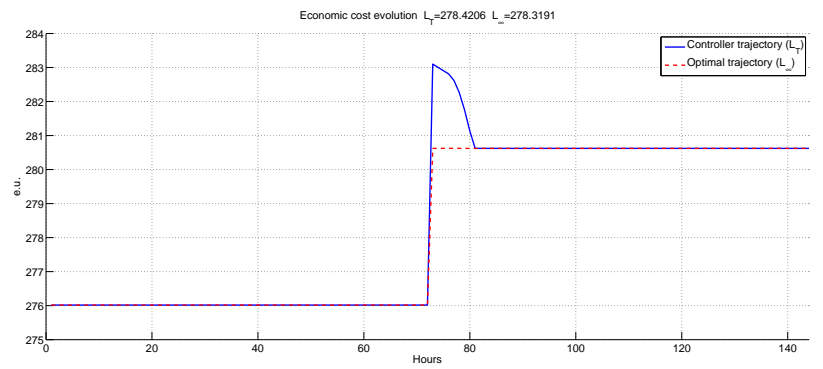

Figure 25: Average economic cost of the optimal trajectory during a period (red) and optimal cost of the controller optimization problem (blue) trajectories.

ically oriented NMPC for cyclic processes. Journal of Process Control 22, $51-59$.

[14] Huang, R., Harinath, E., Biegler, L. T., 2011. Lyapunov stability of economically oriented NMPC for cyclic processes. Journal of Process ControlArticle in press.

[15] Jiang, Z.-P., Wang, Y., 2001. Input-to-state stability for discrete-time nonlinear systems. Automatica 37, 857-869.

[16] Johansson, K. H., 2000. The quadruple-tank process. IEEE Trans. Cont. Sys. Techn. 8, 456-465.

[17] Lee, J., Natarajan, S., Lee, K., 2001. A model-based predictive control approach to repetitive continuous processes with periodic operations. Journal of Process Control 11, 195-207.

[18] Limon, D., Alamo, T., Raimondo, D. M., de la Peña, D. M., Bravo, J. M., Ferramosca, A., Camacho, E. F., 2009. Input-to-state stability: an unifying framework for robust model predictive control. In: Magni, L., Raimondo, D. M., Allgöwer, F. (Eds.), International Workshop on Assessment and Future Direction of Nonlinear Model Predictive Control. Springer, pp. 126.

[19] Limon, D., Alamo, T., Salas, F., Camacho, E. F., 2006. Input to state stability of min-max MPC controllers for nonlinear systems with bounded uncertainties. Automatica 42, 629-645.

[20] Limon, D., Ferramosca, A., Alamo, T., E.F., C., 2012. Model predictive control for changing economic targets. In: Proceedings of 4th IFAC Nonlinear Model Predictive Control Conference International Federation of Automatic Control.

[21] Ocampo-Martinez, C., Puig, V., Cembrano, G., Quevedo, J., 2013. Application of predictive control strategies to the management of complex 
networks in the urban water cycle [applications of control]. IEEE Control Systems Magazine 33 (1), 15-41.

[22] Rawligns, J. B., Amrit, R., 2009. Optimizing process economic performance using model predictive control. In: Magni, L., Raimondo, D. M., Allgöwer, F. (Eds.), International Workshop on Assessment and Future Direction of Nonlinear Model Predictive Control. Springer, pp. 315-323.

[23] Rawlings, J., Angeli, D., Bates, C., 2012. Fundamentals of economic model predictive control. In: Decision and Control (CDC), 2012 IEEE 51st Annual Conference on. pp. 3851-3861.

[24] Rawlings, J. B., Mayne, D. Q., 2009. Model Predictive Control: Theory and Design, 1st Edition. Nob-Hill Publishing.

[25] Rockafellar, R. T., 1970. Convex Analysis. Princeton University Press.

[26] Vidyasagar, M., 1993. Nonlinear Systems Theory, 2nd Edition. PrenticeHall.

[27] Wurth, L., R., H., Marquardt, W., 2011. A two-layer architecture for economically optimal process control and operation. Journal of Process Control 595 $21,311-321$.

[28] Zanin, A. C., de Gouvea, M. T., Odloak, D., 2002. Integrating real time optimization into the model predictive controller of the fcc system. Control Engineering Practice 10, 819-831. 